\title{
GRACEFUL LABELING OF HANGING MODIFIED THETA GRAPHS
}

\author{
G. Sathiamoorthy ${ }^{1 \S}$, T.N. Janakiraman ${ }^{2}$ \\ ${ }^{1}$ Department of Mathematics \\ School of Humanities and Sciences \\ SASTRA University \\ INDIA \\ ${ }^{2}$ Department of Mathematics \\ National Institute of Technology \\ Tiruchirappalli, INDIA
}

\begin{abstract}
Hanging modified theta graphs are obtained from attaching a modified theta graph to each teeth of comb graph $\mathrm{P}_{a_{\mathrm{i}}} \odot \mathrm{L}_{1}$, where $\mathrm{P}_{a_{\mathrm{i}}}$ denotes a path on $\mathbf{a}_{i}$ vertices. In this paper it is proved that symmetric hanging modified theta graphs and non-decreasing symmetric hanging modified theta graphs are graceful.
\end{abstract}

AMS Subject Classification: $05 \mathrm{C} 78$

Key Words: graceful labeling, theta graph, modified theta graph

\section{Introduction}

Graphs considered in this paper are simple finite and undirected. In general $G$ $(\mathrm{V}, \mathrm{E})$ denotes the graph $\mathrm{G}$ with vertex set $\mathrm{V}(\mathrm{G})$ and edge set $\mathrm{E}(\mathrm{G})$, such that $|V(G)|=\mathrm{p},|E(G)|=\mathrm{q}$. A labeling of the vertices of $\mathrm{G}$ with the numbers from 0 to $\mathrm{q}$ is an injective map $\phi: \mathrm{V} \rightarrow\{0,1, . ., q\}$. Let $\phi^{*}: \mathrm{E} \rightarrow\{1,2, \ldots, q\}$ be a bijective. Then a graph $\mathrm{G}$ is graceful if there exists a labeling of its vertices and edges with the condition for all $\mathrm{u}, \mathrm{v} \in \mathrm{V}(\mathrm{G})$ with $\mathrm{uv} \in \mathrm{E}(\mathrm{G})$ such that $\phi^{*}(\mathrm{u} \mathrm{v})=|\phi(u)-\phi(v)|$.

Received: January 20, 2016

Published: April 21, 2016

§ Correspondence author (c) 2016 Academic Publications, Ltd.

url: www.acadpubl.eu 
The concept of graceful labeling was introduced Rosa [2] with the name $\beta$ valuation. Gallian [1] has given the extensive survey of contributions to graceful labeling of variety of graphs.

Definition 1. Theta graph: A theta graph is the union of three internally disjoint paths of length 2 between two vertices.

Definition 2. modified theta graph: A modified theta graph consisting of $n$ internal disjoint paths, in which each path contains $m$ vertices and path end vertices are merged with the vertices $\mathrm{v}_{1}$ and $\mathrm{v}_{2}$ respectively.

\section{Main Result}

Hanging modified theta graphs are results from attaching a modified theta graph to each teeth of comb graph $\mathrm{P}_{a_{i}} \odot \mathrm{L}_{1}$, where $\mathrm{P}_{a_{i}}$ denotes a path on $\mathbf{a}_{i}$ vertices. Designate the $\mathrm{j}^{\text {th }}$ modified theta graph hanging from $\mathrm{b}_{j}$ as $\mathrm{H} \theta\left(\mathrm{n}_{j}, \mathrm{~m}_{j}\right)$ and each $\mathrm{H} \theta\left(\mathrm{n}_{j}, \mathrm{~m}_{j}\right)$ has $\left(\mathrm{m}_{j}-1\right) \mathrm{n}_{j}$ edges. Hanging modified theta graphs satisfy $n \geq 3, m=2 j+1$ and $j=1,2, \ldots, n$. For $j=1,2 n$ may be odd or even and for $\mathrm{j}=3,4, \ldots, \mathrm{n}, \mathrm{n}$ must be odd.

If the internally disjoint paths are not of same length then

$$
\theta_{i}=\theta\left(n_{i}, m_{1}^{l_{1}(i)}, m_{2}^{l_{2}(i)}, \ldots, m_{k}^{l_{k}(i)}\right)
$$

where $l_{k}(i)$ denotes number of internally disjoint paths and $\left(m_{i}\right)$ denotes paths of length $m_{i}-1$ for $\mathrm{i}=1,2, \ldots, \mathrm{k}$, joining $\mathrm{v}_{1}$ and $\mathrm{v}_{2}$ and $\mathrm{l}_{1}(i)+\mathrm{l}_{2}(i)+\ldots$ $+\mathrm{l}_{k}(i)=\mathrm{n}_{i}$. The resultant hanging theta graph deonted by $\mathrm{H}\left(\theta_{1}, \theta_{1}, \ldots, \theta_{n}\right)$ and if all $\mathrm{m}_{j}^{l_{j}(i)}$ are same then it becomes $\mathrm{H} \theta\left(\mathrm{n}_{j}, \mathrm{~m}_{j}\right)$.

Let $\mathrm{n}_{1}, \mathrm{n}_{2}, \ldots, \mathrm{n}_{n}$ be the number of paths contained in each hanging modified theta graph respectively. Let $\mathrm{q}_{1}, \mathrm{q}_{2}, \ldots, \mathrm{q}_{a_{i}}\left(\mathrm{q}_{i}=\mathrm{n}_{i}\left(\mathrm{~m}_{i}-1\right)+1,1 \leq \mathrm{i}\right.$ $\leq \mathrm{a}_{i}$ ) be the number of edges of each hanging modified theta graph from $b_{1}$, $\mathrm{b}_{2}, \ldots, \mathrm{b}_{a}$ respectively. This results $\mathrm{q}=\sum_{d=1}^{a_{i}} q_{d}+\left(\mathrm{a}_{i}-1\right)$. The path vertices in modified theta graph are labeled in the form of $\left(\mathrm{c}_{\left(b a_{i}\right)\left(p a_{i}\right)}^{l e}\right)$ where $\left(\mathrm{ba}_{i}\right)$ denotes base vertex, $\left(\mathrm{pa}_{i}\right)$ denotes internal path index of theta graph and (le) level index.

Condition for symmetric hanging modified theta graphs are all hanging modified theta graphs are isomorphic or at least even number of them must be consecutive.

Condition for non-decreasing symmetric hanging modified theta graphs are 1 . The number of edges in $\mathrm{j}^{\text {th }}$ component and $(\mathrm{j}+1)^{\text {th }}$ component 
of $\mathrm{H} \theta(\mathrm{n}, \mathrm{m})$ must be same, for $\mathrm{j}=1,2, \ldots, \mathrm{n}-1$.

2. The number of paths in each modified theta graph are factors or multiples of factors in relation with the number of edges. Theta's are non-decreasing symmetric denoted as $\mathrm{H} \theta\left(\left(\mathrm{n}_{1}, \mathrm{~m}_{1}\right),\left(\mathrm{n}_{2}, \mathrm{~m}_{2}\right), \ldots,\left(\mathrm{n}_{k}, \mathrm{~m}_{k}\right)\right)$.

Let $\mathrm{E}(\mathrm{A})$ be the collection of base edge $\left(\phi^{*}\left(\mathrm{~b}_{k} \mathrm{~b}_{k+1}\right)\right)$ values, $\mathrm{E}(\mathrm{B})$ be the collection supporting edge $\left(\phi^{*}\left(\mathrm{~b}_{d} \mathrm{t}_{d}\right)\right)$ values for $1 \leq \mathrm{k} \leq \mathrm{a}_{i}-1,1 \leq \mathrm{d} \leq \mathrm{a}_{i}$. Let $\mathrm{E}(\mathrm{D})$ be the collection of first part of modified theta graph edge $\left(\phi^{*}\left(\mathrm{t}_{d} \mathrm{c}_{(d)(i)}^{d}\right)\right)$ values, $\mathrm{E}(\mathrm{F})$ be the collection of intermediary $\operatorname{edge}\left(\phi^{*}\left(\mathrm{c}_{(d)\left(n_{(d-i)}\right)}^{j} \mathrm{c}_{(d)\left(n_{d-i}\right)}^{j+1}\right)\right)$ values and $\mathrm{E}(\mathrm{H})$ be the collection of last part of modified theta graph edge $\left(\phi^{*}\left(\mathrm{v}_{d} \mathrm{c}_{(d)(i)}^{m_{d}}\right)\right)$ values, for $1 \leq \mathrm{d} \leq \mathrm{a}_{i}, 1 \leq \mathrm{i} \leq \mathrm{n}_{d}, 1 \leq \mathrm{j} \leq \mathrm{m}_{d}-3$. Now, $\mathrm{E}(\mathrm{G})=\{\mathrm{E}(\mathrm{A}) \bigcup$ $\mathrm{E}(\mathrm{B}) \cup \mathrm{E}(\mathrm{D}) \cup \mathrm{E}(\mathrm{F}) \cup \mathrm{E}(\mathrm{H})\}$

The hanging modified theta graphs is shown in the following Figure 1.

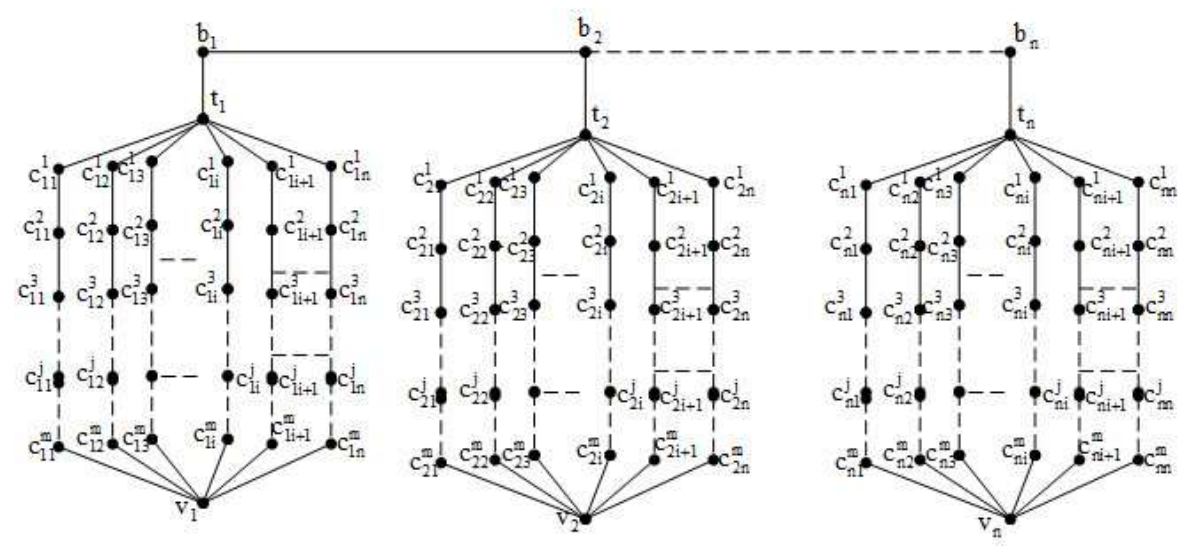

Figure 1: The general form of symmetric or non-decreasing symmetric hanging modified theta graphs

Theorem: $\mathrm{H} \theta\left(\mathrm{n}_{j}, \mathrm{~m}_{j}\right), \mathrm{j}=1,2, \ldots, \mathrm{n}$ is graceful.

Proof. Labeling of vertices in first hanging modified theta graph is as follows :-

Step $1: \phi\left(b_{1}\right)=0, \phi\left(t_{1}\right)=q . \quad \phi\left(v_{1}\right)=\phi\left(t_{1}\right)-\frac{q_{1}-1}{2}$.

Step $2: \phi\left(c_{(1)(1)}^{1}\right)=1, \phi\left(c_{(1)(i)}^{1}\right)=\mathrm{i}, 2 \leq \mathrm{i} \leq \mathrm{n}_{1}$.

Step $3: \phi\left(c_{(1)\left(n_{1}\right)}^{2}\right)=\mathrm{q}-1 . \quad \phi\left(\mathrm{c}_{(1)\left(n_{1}-i\right)}^{2}\right)=\phi\left(\mathrm{c}_{(1)\left(n_{1}+1-i\right)}^{2}\right)-2,1 \leq \mathrm{i} \leq \mathrm{n}_{1}$ -1 . 
Step $4: \phi\left(c_{(1)(1)}^{2 j+1}\right)=1+\mathrm{n}_{1} \mathrm{j}, 1 \leq \mathrm{j} \leq \frac{m_{1}-3}{2}$.

Step $5: \phi\left(c_{(1)(i)}^{2 j+1}\right)=\phi\left(c_{(1)(i-1)}^{2 j+1}\right)+1,2 \leq \mathrm{i} \leq \mathrm{n}_{1}, 1 \leq \mathrm{j} \leq \frac{m_{1}-3}{2}$.

Step $6: \phi\left(\mathrm{c}_{(1)(n)}^{2 j+2}\right)=\mathrm{q}-1-\mathrm{n}_{1} \mathrm{j}, 1 \leq \mathrm{j} \leq \frac{m_{1}-5}{2}$.

Step $7: \phi\left(\mathrm{c}_{(1)(n-i)}^{2 j+2}\right)=\phi\left(\mathrm{c}_{(1)(n+1-i)}^{2 j+2}\right)-2,1 \leq \mathrm{i} \leq \mathrm{n}_{1}-1,1 \leq \mathrm{j} \leq \frac{m_{1}-5}{2}$.

Now, labeling of vertices in second hanging modified theta graph has given as under.

Step $1: \phi\left(b_{2}\right)=q-\left(q_{1}+1\right), \phi\left(t_{2}\right)=q_{1}+1 . \phi\left(v_{2}\right)=\phi\left(t_{2}\right)-\frac{q_{2}-1}{2}$.

Step $2: \phi\left(c_{(2)(1)}^{1}\right)=\phi\left(b_{2}\right)+1, \phi\left(c_{(2)(i)}^{1}\right)=\phi\left(b_{2}\right)+\mathrm{i}, 2 \leq \mathrm{i} \leq \mathrm{n}_{2}$.

Step $3: \phi\left(\mathrm{c}_{(2)\left(n_{2}\right)}^{2}\right)=\phi\left(\mathrm{t}_{2}\right)-1 . \quad \phi\left(\mathrm{c}_{(2)\left(n_{2}-i\right)}^{2}\right)=\phi\left(\mathrm{c}_{(2)\left(n_{2}+1-i\right)}^{2}\right)-2,1 \leq \mathrm{i} \leq$ $\mathrm{n}_{2}-1$.

Step $4: \phi\left(\mathrm{c}_{(2)(1)}^{2 j+1}\right)=\phi\left(\mathrm{c}_{(2)(1)}^{1}\right)+\mathrm{n}_{2} \mathrm{j}, 1 \leq \mathrm{j} \leq \frac{m_{2}-3}{2}$.

Step $5: \phi\left(c_{(2)(i)}^{2 j+1}\right)=\phi\left(c_{(2)(i-1)}^{2 j+1}\right)+1,2 \leq \mathrm{i} \leq \mathrm{n}_{2}, 1 \leq \mathrm{j} \leq \frac{m_{2}-3}{2}$.

Step $6: \phi\left(c_{(2)(n)}^{2 j+2}\right)=\phi\left(c_{(2)\left(n_{2}\right)}^{2}\right)-\mathrm{n}_{2} \mathrm{j}, 1 \leq \mathrm{j} \leq \frac{m_{2}-5}{2}$.

Step $7: \phi\left(\mathrm{c}_{(2)(n-i)}^{2 j+2}\right)=\phi\left(\mathrm{c}_{(2)(n+1-i)}^{2 j+2}\right)-2,1 \leq \mathrm{i} \leq \mathrm{n}_{2}-1,1 \leq \mathrm{j} \leq \frac{m_{2}-5}{2}$.

(Let $\mathrm{a}_{i}=2 \lambda+1$ for odd and $\mathrm{a}_{i}=2 \lambda$ for even)

Labeling of vertices in odd segments of hanging modified theta graphs are as follows :-

Step $1: \phi\left(b_{2 d+1}\right)=\phi\left(b_{2 d-1}\right)+\mathrm{q}_{2 d}+1,1 \leq \mathrm{d} \leq \lambda$, for $\mathrm{n}$ is odd, $1 \leq \mathrm{d} \leq$ $\lambda-1$, for $\mathrm{n}$ is even.

$\phi\left(\mathrm{t}_{2 d+1}\right)=\mathrm{q}-\phi\left(\mathrm{b}_{2 d+1}\right), 1 \leq \mathrm{d} \leq \lambda$, for $\mathrm{n}$ is odd, $1 \leq \mathrm{d} \leq \lambda-1$, for $\mathrm{n}$ is even.

$\phi\left(\mathrm{v}_{2 d+1}\right)=\phi\left(\mathrm{t}_{2 d+1}\right)-\frac{q_{2 d+1}-1}{2}, 1 \leq \mathrm{d} \leq \lambda$, for $\mathrm{n}$ is odd, $1 \leq \mathrm{d} \leq \lambda-1$, for $\mathrm{n}$ is even.

Step $2: \phi\left(\mathrm{c}_{(2 d+1)(1)}^{1}\right)=\phi\left(\mathrm{b}_{2 d+1}\right)+1 . \quad \phi\left(\mathrm{c}_{(2 d+1)(i)}^{1}\right)=\phi\left(\mathrm{b}_{2 d+1}\right)+\mathrm{i}, 2 \leq \mathrm{i} \leq$ $\mathrm{n}_{2 d+1}, 1 \leq \mathrm{d} \leq \lambda$, for $\mathrm{n}$ is odd, $1 \leq \mathrm{d} \leq \lambda-1$, for $\mathrm{n}$ is even.

Step 3: $\quad \phi\left(\mathrm{c}_{(2 d+1)\left(n_{2 d+1}\right)}^{2}\right)=\phi\left(\mathrm{t}_{2 d+1}\right)-1 . \quad \phi\left(\mathrm{c}_{(2 d+1)\left(n_{2 d+1}-i\right)}^{2}\right)=$ $\phi\left(\mathrm{c}_{(2 d+1)\left(n_{2 d+1}+1-i\right)}^{2}\right)-2,1 \leq \mathrm{i} \leq \mathrm{n}_{2 d+1}-1,1 \leq \mathrm{d} \leq \lambda$, for $\mathrm{n}$ is odd, $1 \leq \mathrm{d} \leq \lambda-1$, for $\mathrm{n}$ is even. 
Step $4: \phi\left(c_{(2 d+1)(1)}^{2 j+1}\right)=\phi\left(c_{(2 d+1)(1)}^{1}\right)+n_{2 d+1} \mathrm{j}, 1 \leq \mathrm{j} \leq \frac{m_{2 d+1}-3}{2}, 1 \leq \mathrm{d} \leq \lambda$, for $\mathrm{n}$ is odd, $1 \leq \mathrm{d} \leq \lambda-1$, for $\mathrm{n}$ is even.

Step 5: $\phi\left(\mathrm{c}_{(2 d+1)(i)}^{2 j+1}\right)=\phi\left(\mathrm{c}_{(2 d+1)(i-1)}^{2 j+1}\right)+1,2 \leq \mathrm{i} \leq \mathrm{n}_{2 d+1}, 1 \leq \mathrm{j} \leq \frac{m_{2 d+1}-3}{2}$, $1 \leq \mathrm{d} \leq \lambda$, for $\mathrm{n}$ is odd, $1 \leq \mathrm{d} \leq \lambda-1$, for $\mathrm{n}$ is even.

Step $6: \phi\left(\mathrm{c}_{(2 d+1)(n)}^{2 j+2}\right)=\phi\left(\mathrm{t}_{2 d+1}\right)-\mathrm{n}_{2 d+1} \mathrm{j}, 1 \leq \mathrm{j} \leq \frac{m_{2 d+1}-5}{2}, 1 \leq \mathrm{d} \leq \lambda$, for $\mathrm{n}$ is odd, $1 \leq \mathrm{d} \leq \lambda-1$, for $\mathrm{n}$ is even.

Step $7: \phi\left(\mathrm{c}_{(2 d+1)(n-i)}^{2 j+2}\right)=\phi\left(\mathrm{c}_{(2 d+1)(n+1-i)}^{2 j+2}\right)-2,1 \leq \mathrm{i} \leq \mathrm{n}_{2 d+1}-1,1 \leq \mathrm{j} \leq$ $\frac{m_{2 d+1}-5}{2}, 1 \leq \mathrm{d} \leq \lambda$, for $\mathrm{n}$ is odd, $1 \leq \mathrm{d} \leq \lambda-1$, for $\mathrm{n}$ is even.

The labeling of vertices in even segments of hanging modified theta graphs has given as under.

Step $1: \phi\left(\mathrm{b}_{2 d+2}\right)=\phi\left(\mathrm{b}_{2 d}\right)-\left(\mathrm{q}_{2 d+1}+1\right) . \phi\left(\mathrm{t}_{2 d+2}\right)=\phi\left(\mathrm{t}_{2 d}\right)+\mathrm{q}_{2 d+1}+1$. $\phi\left(\mathrm{v}_{2 d+2}\right)=\phi\left(\mathrm{t}_{2 d+2}\right)-\frac{q_{2 d+2}-1}{2}, 1 \leq \mathrm{d} \leq \lambda-1$.

Step $2: \phi\left(\mathrm{c}_{(2 d+2)(1)}^{1}\right)=\phi\left(\mathrm{b}_{2 d+2}\right)+1$.

$\phi\left(\mathrm{c}_{2 d+2 i}^{1}\right)=\phi\left(\mathrm{b}_{2 d+2}\right)+\mathrm{i}, 2 \leq \mathrm{i} \leq \mathrm{n}_{2 d+2}, 1 \leq \mathrm{d} \leq \lambda-1$.

Step $3: \quad \phi\left(\mathrm{c}_{(2 d+2)\left(n_{2 d+2}\right)}^{2}\right)=\phi\left(\mathrm{t}_{2 d+2}\right)-1 . \quad \phi\left(\mathrm{c}_{(2 d+2)\left(n_{2 d+2}-i\right)}^{2}\right)=$ $\phi\left(\mathrm{c}_{(2 d+2)\left(n_{2 d+2}+1-i\right)}^{2}\right)-2,1 \leq \mathrm{i} \leq \mathrm{n}_{2 d+2}-1,1 \leq \mathrm{d} \leq \lambda-1$.

Step $4: \phi\left(\mathrm{c}_{(2 d+2)(1)}^{2 j+1}\right)=\phi\left(\mathrm{c}_{(2 d+2)(1)}^{1}\right)+\mathrm{n}_{2 d+2} \mathrm{j}, 1 \leq \mathrm{j} \leq \frac{m_{2 d+2}-3}{2}, 1 \leq \mathrm{d} \leq$ $\lambda-1$.

Step 5: $\phi\left(\mathrm{c}_{(2 d+2)(i)}^{2 j+1}\right)=\phi\left(\mathrm{c}_{(2 d+2)(i-1)}^{2 j+1}\right)+1,2 \leq \mathrm{i} \leq \mathrm{n}_{2 d+2}, 1 \leq \mathrm{j} \leq \frac{m_{2 d+2}-3}{2}$, $1 \leq \mathrm{d} \leq \lambda-1$.

Step $6: \phi\left(\mathrm{c}_{(2 d+2)(n)}^{2 j+2}\right)=\phi\left(\mathrm{c}_{(2 d+2)\left(n_{2 d+2}\right)}^{2}\right)-\mathrm{n}_{2 d+2} \mathrm{j}, 1 \leq \mathrm{j} \leq \frac{m_{2 d+2}-5}{2}, 1 \leq \mathrm{d} \leq$ $\lambda-1$.

Step $7: \phi\left(\mathrm{c}_{(2 d+2)(n-i)}^{2 j+2}\right)=\phi\left(\mathrm{c}_{(2 d+2)(n+1-i)}^{2 j+2}\right)-2,1 \leq \mathrm{i} \leq \mathrm{n}_{2 d+2}-1,1 \leq \mathrm{j} \leq$ $\frac{m_{2 d+2}-5}{2}, 1 \leq \mathrm{d} \leq \lambda-1$.

Now, induced labeling of edge values are as follows:-

$1: \phi^{*}\left(\mathrm{~b}_{1} \mathrm{t}_{1}\right)=\mathrm{q} \cdot[2]: \phi^{*}\left(\mathrm{t}_{1} \mathrm{c}_{(1)(i)}^{1}\right)=\mathrm{q}-\mathrm{i}, 1 \leq \mathrm{i} \leq \mathrm{n}_{1}$.

3: $\phi^{*}\left(\mathrm{c}_{(1)(n+1-i)}^{j} \mathrm{c}_{(1)(n+1-i)}^{j+1}\right)=\mathrm{q}-\mathrm{jn}_{1}-\mathrm{i}, 1 \leq \mathrm{i} \leq \mathrm{n}_{1}, 1 \leq \mathrm{j} \leq \mathrm{m}_{1}-3$.

$4: \phi^{*}\left(\mathrm{v}_{1} \mathrm{c}_{(1)(i)}^{m_{1}-2}\right)=\mathrm{q}-\left(\mathrm{m}_{1}-2\right) \mathrm{n}_{1}-\mathrm{i}, 1 \leq \mathrm{i} \leq \mathrm{n}_{1}$. 
$5: \phi^{*}\left(b_{1} b_{2}\right)=q-q_{1} \cdot[6]: \phi^{*}\left(v_{2} c_{(2)\left(n_{2}+1-i\right)}^{m_{2}-2}\right)=\mathrm{q}-\mathrm{q}_{1}-\mathrm{i}, 1 \leq \mathrm{i} \leq \mathrm{n}_{2}$.

$7: \phi^{*}\left(\mathrm{c}_{(2)(i)}^{m_{2}-j} \mathrm{c}_{(2)(i)}^{m_{2}-(j+1)}\right)=\mathrm{q}-\mathrm{q}_{1}-(\mathrm{j}-2) \mathrm{n}_{2}-\mathrm{i}, 1 \leq \mathrm{i} \leq \mathrm{n}_{2}, 2 \leq \mathrm{j} \leq \mathrm{m}_{2}-3$.

$8: \phi^{*}\left(\mathrm{t}_{2} \mathrm{c}_{(2)(i)}^{1}\right)=\mathrm{q}-\left(\mathrm{q}_{1}+\mathrm{q}_{2}\right)+\mathrm{n}_{2}+1-\mathrm{i}, 1 \leq \mathrm{i} \leq \mathrm{n}_{2}$.

$9: \phi^{*}\left(\mathrm{~b}_{2} \mathrm{t}_{2}\right)=\mathrm{q}-\left(\mathrm{q}_{1}+\mathrm{q}_{2}\right) \cdot[\mathbf{1 0}]: \phi^{*}\left(\mathrm{~b}_{2} \mathrm{~b}_{3}\right)=\mathrm{q}-\left(\mathrm{q}_{1}+\mathrm{q}_{2}\right)-1$.

Odd indexed base vertices are termed as odd segments of $\mathrm{H} \theta\left(\mathrm{n}_{j}, \mathrm{~m}_{j}\right)$. Similarly, from the even indexed base vertices are termed as even segments of $\mathrm{H} \theta\left(\mathrm{n}_{j}, \mathrm{~m}_{j}\right)$.

Now, induced edge values of odd segments are as follows :-

$11: \phi^{*}\left(\mathrm{~b}_{2 d} \mathrm{~b}_{2 d+1}\right)=\mathrm{q}-\sum_{k=1}^{2 d} q_{k}-\mathrm{d}, 1 \leq \mathrm{d} \leq \lambda$, for a is odd, $1 \leq \mathrm{d} \leq \lambda-1$, for a is even.

$12: \phi^{*}\left(\mathrm{~b}_{2 d+1} \mathrm{t}_{2 d+1}\right)=\mathrm{q}-\sum_{k=1}^{2 d} q_{k}-2 \mathrm{~d}, 1 \leq \mathrm{d} \leq \lambda$, for a is odd, $1 \leq \mathrm{d} \leq \lambda-1$, for $\mathrm{a}$ is even.

$13: \phi^{*}\left(\mathrm{t}_{2 d+1} \mathrm{c}_{(2 d+1)(i)}^{2 d+1}\right)=\mathrm{q}-\mathrm{i}-\sum_{k=1}^{2 d} q_{k}-2 \mathrm{~d}, 1 \leq \mathrm{d} \leq \lambda$, for a is odd, $1 \leq \mathrm{d} \leq$ $\lambda-1$, for a is even, $1 \leq \mathrm{i} \leq \mathrm{n}_{1}$.

$14: \phi^{*}\left(\mathrm{c}_{(2 d+1)\left(\left(n_{2 d+1}\right)+1-i\right)}^{j} \mathrm{c}_{(2 d+1)\left(\left(n_{2 d+1}\right)+1-i\right)}^{j+1}\right)=\mathrm{q}-\mathrm{j} \mathrm{n}_{2 d+1}-\mathrm{i}-\sum_{k=1}^{2 d} q_{k}-2 \mathrm{~d}$, $1 \leq \mathrm{d} \leq \lambda$, for a is odd, $1 \leq \mathrm{d} \leq \lambda-1$, for a is even, $1 \leq \mathrm{i} \leq \mathrm{n}_{2 d+1}, 1 \leq \mathrm{j}$ $\leq \mathrm{m}_{2 d+1}-3$.

$15: \phi^{*}\left(\mathrm{v}_{2 d+1} \mathrm{c}_{(2 d+1)(i)}^{m_{2 d+1}-2}\right)=\mathrm{q}-\left(\mathrm{m}_{2 d+1}-2\right) \mathrm{n}_{2 d+1}-\sum_{k=1}^{2 d} q_{k}-2 \mathrm{~d}, 1 \leq \mathrm{d} \leq \lambda$, for a is odd, $1 \leq \mathrm{d} \leq \lambda-1$, for a is even, $1 \leq \mathrm{i} \leq \mathrm{n}_{2 d+1}$.

$\phi^{*}\left(\mathrm{~b}_{2 d+1} \mathrm{~b}_{2 d+2}\right)=\mathrm{q}-\sum_{k=1}^{2 d+1} q_{k}-2 \mathrm{~d}, 1 \leq \mathrm{d} \leq \lambda-1$.

Induced edge values of even segments are as follows :-

$16: \phi^{*}\left(\mathrm{v}_{2 d+2} \mathrm{c}_{(2 d+2)\left(n_{2 d+2}+1-i\right)}^{m_{2 d+2}-2}\right)=\mathrm{q}-\sum_{k=1}^{2 d+1} q_{k}-2 \mathrm{~d}-\mathrm{i}, 1 \leq \mathrm{d} \leq \lambda-1,1 \leq \mathrm{i} \leq$ $\mathrm{n}_{2 d+2}$. 
$17: \phi^{*}\left(\mathrm{c}_{(2 d+2)(i)}^{m_{2 d+2}-j} \mathrm{c}_{(2 d+2)(i)}^{m_{2 d+2}-(j+1)}\right)=\mathrm{q}-\sum_{k=1}^{2 d+1} q_{k}-2 \mathrm{~d}-1-(\mathrm{j}-1) \mathrm{n}_{2 d+2}-\mathrm{i}, 1 \leq$ $\mathrm{i} \leq \mathrm{n}_{2 d+2}, 2 \leq \mathrm{j} \leq \mathrm{m}_{2 d+2}-3,1 \leq \mathrm{d} \leq \lambda-1$.

$18: \phi^{*}\left(\mathrm{t}_{2 d+2} \mathrm{c}_{(2 d+2)\left(n_{2 d+2}+1-i\right)}^{1}\right)=\mathrm{q}-\sum_{k=1}^{2 d+2} q_{k}-2 \mathrm{~d}+1+\mathrm{n}_{2 d+2}-\mathrm{i}, 1 \leq \mathrm{i} \leq$ $\mathrm{n}_{2 d+2}, 1 \leq \mathrm{d} \leq \lambda-1$.

$19: \phi^{*}\left(\mathrm{~b}_{2 d+2} \mathrm{t}_{2 d+2}\right)=\mathrm{q}-\sum_{k=1}^{2 d+2} q_{k}-2 \mathrm{~d}, 1 \leq \mathrm{d} \leq \lambda-1$.

$20: \phi^{*}\left(\mathrm{~b}_{2 d+2} \mathrm{~b}_{2 d+3}\right)=\mathrm{q}-\sum_{k=1}^{2 d+2} q_{k}-2 \mathrm{~d}-1,1 \leq \mathrm{d} \leq \lambda-1$.

The resultant edge values $\mathrm{E}(\mathrm{G})$ are distinct.

Result: The hanging modified theta graphs $\mathrm{H} \theta\left(\mathrm{n}_{j}, \mathrm{~m}_{j}\right), \mathrm{m}=2 \mathrm{j}+1$ and $\mathrm{j}=$ $1,2, \ldots, \mathrm{n}$ which includes symmetric modified theta graphs and non-decreasing symmetric modified theta graphs are graceful.

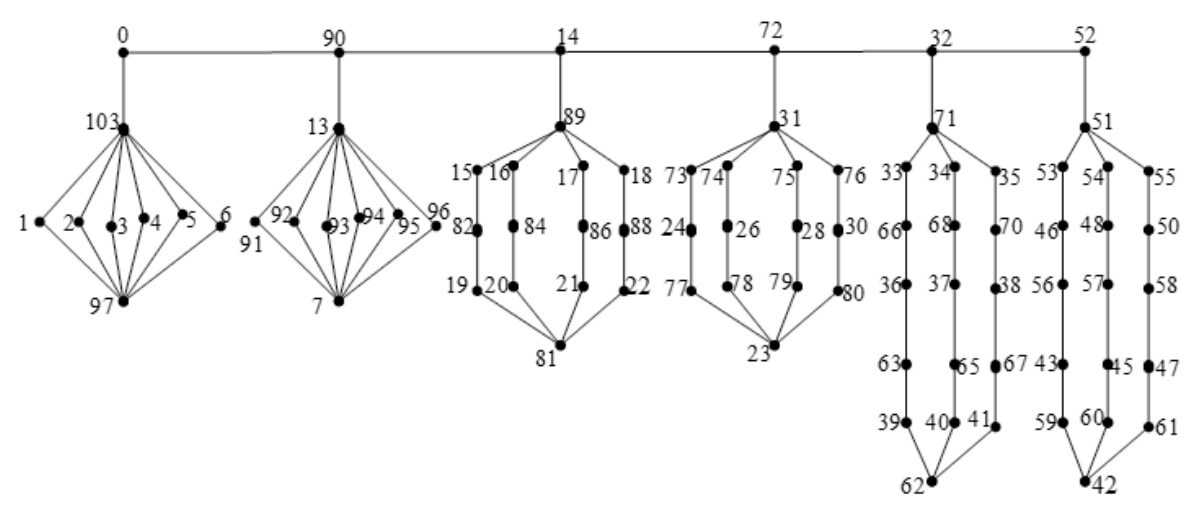

Figure 2: Graceful labeling of $\mathrm{H} \theta\left(6 \mathrm{~m}_{3}^{1,2}, 4 \mathrm{~m}_{5}^{3,4}, 3 \mathrm{~m}_{7}^{5,6}\right)$

Example for hanging non-decreasing symmetric modified theta graphs are shown below. 


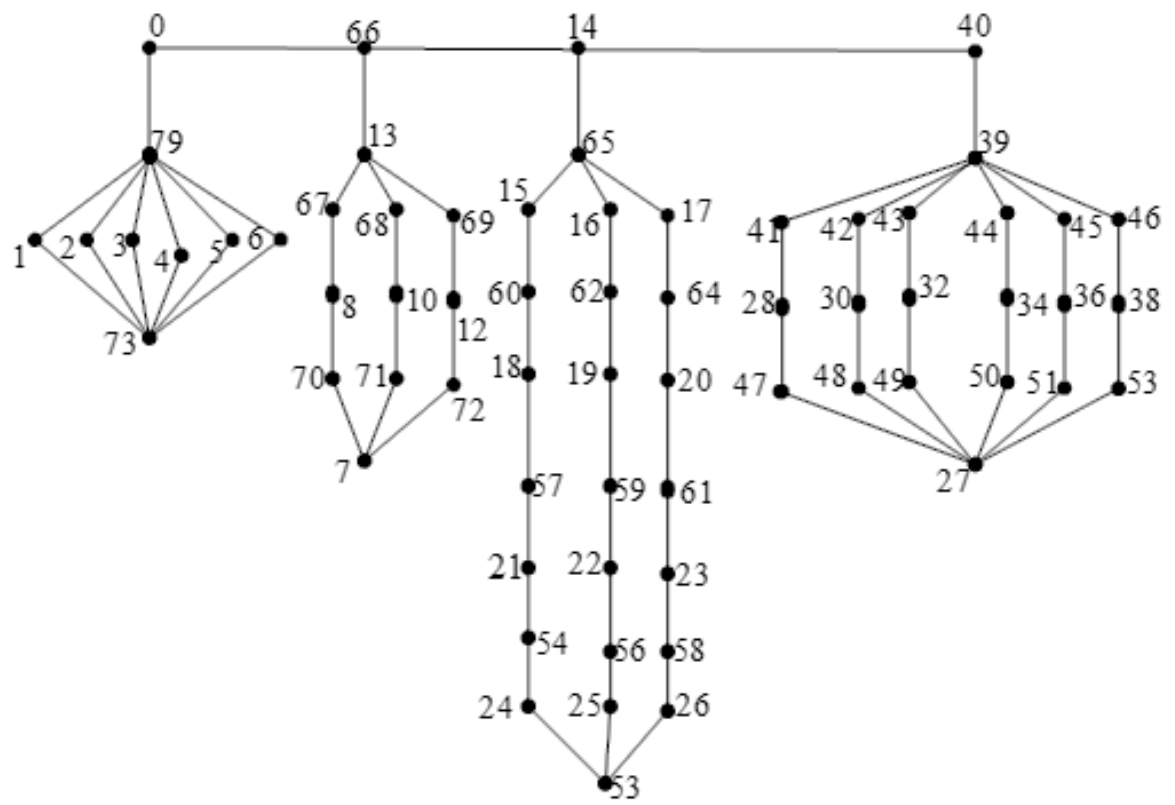

Figure 3: Graceful labeling of $\mathrm{H} \theta\left(6 \mathrm{~m}_{3}^{1}, 3 \mathrm{~m}_{5}^{2}, 3 \mathrm{~m}_{9}^{3}, 6 \mathrm{~m}_{5}^{4}\right)$

\section{References}

[1] Joseph A. Gallian, A Dynamic Survey of Graceful Labeling, The Electronic Journal Combinatorics, 18 (2011).

[2] A. Rosa, On certain valuations of the vertices of a graph. Theory of graphs, In: Internat. Symposium, Rome, July 1966, Gordon and Breach, N.Y.and Dunod Paris (1967), 349355.

[3] G. Sathiamoothy, T.N. Janakiraman, Modified theta graphs are graceful (Communication). 